\title{
Clinicomicrobiological profile of burn wound infections and antibiogram of the isolates in a Tertiary Care Hospital, Karnataka
}

\author{
Sumaira Qayoom ${ }^{1}$, Mariraj Jeer², Muzaffar Amin ${ }^{3, *}$, Talat Masoodi', Syed Khurshid ${ }^{5}$ \\ ${ }^{1,3}$ Senior Resident, ${ }^{4}$ Lecturer, ${ }^{5}$ Professor \& Head, SKIMS Medical College and Hospital, Srinagar, Jammu \& Kashmir, \\ ${ }^{2}$ Professor, Vijayanagara Institute of Medical Sciences, Ballari, Karnataka, India
}

*Corresponding Author:

Email: sumairabeigh@gmail.com

\begin{abstract}
Objective: To find out the bacteriological profile of post burn infections in wound.

Place and duration of study: Vijayanagar institute of medical sciences, ballari, over a period of one year from $1^{\text {st }}$ January $2014-$ $31^{\text {st }}$ December 2014.

Study design: Prospective

Materials and Methods: Swabs were collected from the burn wounds using standard methods and cultured for the growth of the bacteria.The organisms isolated were identified by standard microbiological methods following which antimicrobial susceptibility test was done by Kirby-baeur disk diffusion test.

Results: The predominant organism isolated from swab culture was Pseudomonas aeruginosa(23\%), followed by Staphylococcus aureus(22\%), E.coli(14\%), K.pneumoniae(9\%), K.oxytoca(8\%), CONS(7\%), Proteus mirabilis(5\%), C.freundii(4\%) and C.koseri(3\%),others(3\%) and sterile culture(3\%).The antibiogram of Pseudomonas aeruginosa showed high susceptibility to Imipenem( 81\%) Piperacillin/Tazobactam (78\%), Amikacin(76\%), Ceftazidime(60\%) and low susceptibility to Gentamicin(57\%), Tobramycin(57\%), Cefotaxime(47\%), and Ceftriaxone(39\%).Total resistance pattern was noted in Ampicillin, Cotrimoxazole, Ciprofloxacin.
\end{abstract}

Keywords: Antibiotic susceptibility, Burn wounds, Pseudomonas aeruginosa.

\section{Introduction}

After road traffic accidents, the second major cause of trauma related deaths is burns. ${ }^{1}$ Around 200,000 deaths account to burns every year in India. ${ }^{2}$ Almost $95 \%$ of global burn deaths and disabilities are estimated to occur in low and middle income countries of the world. The microbial colonization of the wound is due to loss of the natural cutaneous barrier (skin) and coagulated proteins in the avascular burn wound.

Colonization, in some patients, is followed by the invasion of microorganisms, giving rise to burn wound infection. The host defence mechanisms are impaired by severe thermal injury causing increased susceptibility to infection, not only by common human pathogens, but also by organisms not normally pathogenic in the uncompromised host. ${ }^{4,5}$

Burns become infected because the environment at the site of the wound is ideal for the multiplication of the infecting organism. ${ }^{6}$ The common pathogens isolated from a burn wound worldwide are Staphylococcus aureus-20-50\%, Pseudomonas aeruginosa-20-25\%, Candida species-3\% and other bacteria and fungi -less than $1 \% .4,6,8$

In Indian centers, Pseudomonas species was the predominant isolate followed by Staphylococcus aureus, Klebsiella species, Enterococcus species and Acinetobacter species. ${ }^{51,52}$

Factors like nature of burn injury itself, immunocompromised status of the patient, invasive diagnostic and therapeutic procedures and prolonged ICU stay increase the likelihood of nosocomial infections in burn patients.Because the spectrum of bacterial isolates varies with time and geographical areas therefore periodic review of the isolation pattern and antibiogram of the burn ward is necessary, which forms the basis for modification of drug regimen strategy. Therefore, the present study was planned to determine the bacteriological pattern from the burn wound infections over a period of one year, and to determine the antimicrobial resistance of the isolates.

\section{Material and Methods}

The present work includes investigation of 100 post burn infection cases admitted in burn ward of VIMS, Ballari over a period of one year. Wound swabs were collected aseptically and brought to the laboratory. Swabs were inoculated onto following media for aerobic bacterial cultures: Blood agar, MacConkey's agar, Chocolate agar. The inoculated media were incubated aerobically at $37^{\circ} \mathrm{C}$ for $24 \mathrm{hrs}$. In case of no growth after $24 \mathrm{hrs}$, the plates were further incubated for another $24 \mathrm{hrs}$. The sample was also put into liquid media (BHI broth) and was subcultured after overnight incubation. Organisms grown were then identified on the basis of their microscopic morphology, Gram's staining characteristics, colonial and cultural characteristics, and biochemical reactions as per the standard protocol using standard laboratory procedures. The antibiotic sensitivity of aerobic bacterial isolates was performed on Mueller Hinton agar plates by standardized Kirby Bauer disc diffusion technique as per the CLSI guidelines. The antimicrobial discs were 
obtained from Hi Media Laboratories Private Limited, Mumbai. The quality control for antimicrobial susceptibility testing was done with standard strains of E.coli (ATCC 25922), S. aureus (ATCC 25923) and Ps. aeruginosa (ATCC 27853). The drugs used for Gram positive organisms were:

Ampicillin (Amp) - $10 \mu \mathrm{g}$, Cefoxitin (Cef) - $30 \mu \mathrm{g}$, Erythromycin (Ery) - $15 \mu \mathrm{g}$,

Clindamycin (Cd) - $2 \mu \mathrm{g}$, Co-trimoxazole (Cot) - $25 \mu \mathrm{g}$

Doxycycline (Do) $-30 \mu \mathrm{g}$

Linezolid (Li) -30 $\mu \mathrm{g}$, Vancomycin (Va) $-30 \mu \mathrm{g}$,

Levofloxacin (Lf) $-5 \mu \mathrm{g}$,

Gentamicin (Gen) $-10 \mu \mathrm{g}$. The drugs used for Gram negative organisms were:

Ampicillin (Amp) - $10 \mu \mathrm{g}$, Amoxicillin-clavulanic acid (Amc) - $20 \mu \mathrm{g} / 10 \mu \mathrm{g}$, Amikacin (Ak) $-30 \mu \mathrm{g}$, Gentamicin (Gen) -10 $\mu \mathrm{g}$, Tobramycin (Tob) - $10 \mu \mathrm{g}$, Ceftriaxone (Ctr) - $30 \mu \mathrm{g}$ Cefotaxime (Ctx) $-30 \mu \mathrm{g}$ Ceftazidime (Caz) - $30 \mu \mathrm{g}$, Ciprofloxacin (Cip) - $5 \mu \mathrm{g}$, Co-trimoxazole (Cot) $-25 \mu \mathrm{g}$ Piperacillin (Pip) - $100 \mu \mathrm{g}$, Piperacillin-tazobactum (Pip/Taz) $\quad-100 / 10 \quad \mu g$ Aztreonam (Az) - $30 \mu \mathrm{g}$, Imipenem (Im) - 10 $\mu \mathrm{g}$.

\section{Results}

The most severe types of injuries suffered by the human body are burn injuries with an attendant high mortality and morbidity rate and form a major public health problem. ${ }^{8,5}$ Since each population seems to have its own epidemiological characteristics and knowledge of the epidemiology of burns is needed to select target groups for preventive action, therefore epidemiological studies are a prerequisite for effective burn prevention programs. ${ }^{8,6}$ Out of 100 cases of burn wound infections, 52 were females $(52 \%)$ and 48 were males $(48 \%)$.The ratio of females to males was $1.45: 1$. The reason could be because women spend more time in kitchen thereby exposing themselves to increased risks of fire in addition to their clothing style which comprises of flowing dupatta or sari. ${ }^{1}$ This finding is in accordance with similar studies conducted by Neha Chauhan et al, ${ }^{1}$ Gowri Shankar et $\mathrm{al}^{3}$ and by Sumit Dutt Bhardwaj et $\mathrm{al}^{9,4}$ who reported female preponderance of burn wound infections. Burn wound infections were most common in $21-40$ years of age group $(60 \%)$, followed by $1-20$ years agegroup $(26 \%)$ and $41-60$ year age group $(13 \%)$. The least affected age group was above 60(1\%).This correlates with the study done by Neha Chauhan et al, ${ }^{1}$ Gowri Shankar et al, ${ }^{3}$ Anil Batra ${ }^{8,6}$ who reported about $67-70 \%$ cases of burns were between 20-40 years of age, suggesting that this age group is most vulnerable to burn injuries. Only one patient was older than 60 years of age according to the study done by Neha Chauhan, ${ }^{1}$ which is consistent with the findings of the current study. Out of 100 cases, 32 cases(32\%) acquired 21$30 \%$ TBSA burns,30 cases(30\%) between $41-50 \%$ TBSA burns, followed by 19 cases $(19 \%)$ between 31$40 \%$.Least number of cases were between $61-70 \%$ i.e 1 case $(1 \%)$ followed by 7 cases $(7 \%)$ between $51-60 \%$
TBSA burns. These findings are at par with a study conducted by Md Sohaib Akhtar et al, ${ }^{8,7}$ wherein maximum number of burn cases fall between $20-40 \%$ TBSA burns and least number of cases(20\%) are with TBSA above $40 \%$. Degree of burns is directly proportional to the incidence of burn wound sepsis and subsequent mortality. Out of 100 cases, 70 cases had second degree burns(70\%), followed by 27 cases who had first degree burns(27\%) and only 3 cases had third degree burns(3\%). These findings are similar to the study done by Md Sohaib et al, ${ }^{8,7}$ wherein first degree burns constituted the most common type of burn injury and the least common was fourth degree burn. This is in contrast to the study done by Tahir Saleem Khan et al, ${ }^{9}$ wherein maximum number of cases( $22 \%$ ) had third degree burns and only $2 \%$ cases had first degree burn. Out of 116 isolates, 34 samples $(29.31 \%)$ were gram positive and $82(70.69 \%$ ) were gram negative organisms. Shobha Chamania et $\mathrm{al}^{8}$ and Manjula Mehta et $\mathrm{al}^{4,8}$ in their studies also found Gram negative organisms $(75 \%$ and $65 \%$ respectively) to be the predominant isolates, followed by Gram positive organisms. Out of 100 samples, 84 yielded single isolates and 16 yielded multiple isolates. Pseudomonas aeruginosa $(23 \%)$ was the predominant organism isolated followed by Staphylococcus aureus $(22 \%)$, E.coli(14\%), K.pneumoniae (9\%), K.oxytoca $(8 \%)$, CONS(7\%), Proteusmirabilis(5\%), C.freundii(4\%) and C.koseri(3\%). Out of 116 isolates, 2 were of Acinetobacter baumanii and one was identified as Nonfermenting GNB.Only 3 samples out of 100 yielded No Growth on culture.

Manjula Mehta et $\mathrm{al}^{48}$,Shobha Chamania et $\mathrm{al},{ }^{8}$ Sarita Mohapatra et $\mathrm{al}^{9,2}$ also found in their studies Pseudomonas aeruginosa to be the predominating organism(52\%,43\%, and $31 \%$ and respectively) infecting the burn wounds, same findings were confirmed in study done by Ernest $\mathrm{A}$ et $\mathrm{al}^{89}$ in United Kingdom and Iman A Hussein et $\mathrm{al}^{5,8}(58.3 \%)$ in. Drug susceptibility test shows high suceptibility of Pseudomonas for Imipenem( $81 \%)$, Piperacillintazobactam(78\%), Amikacin(76\%), Ceftazidime(60\%) and low susceptibility for Gentamicin(57\%), Tobramycin(57\%), Ceftriaxone(39\%), Cefotaxime (47\%).Total resistance pattern was noted in Ampicillin, Cotrimoxazole, Ciprofloxacin. On the other hand, Pseudomonas was found to be more sensitive to newer antimicrobials like imipenem(only $4.5 \%$ resistance), followed by Ceftazidime/clavulanic $\operatorname{acid}(21.8 \%)$ and Cefoperazone/sulbactam (25.67\%). This could be because of the reason that these reserve drugs are used as last options for multi-drug resistant bacteria in our hospital settings. This study coincides with the study done in Baghdad by Iman A Hussein et $\mathrm{al}^{5,8}$ wherein Pseudomonas has shown $100 \%$ resistance to Ampicillin and Cotrimoxazole. Also, pseudomonas isolates have shown low level of resistance to Amikacin, Tobramycin and Gentamicin. The resistance 
is high for third generation cephalosporins like Ceftriaxone(61\%), Ceftazidime(40\%) and Cefotaxime(53\%). Staphylococcus aureus showed total sensitivity to Vancomycin and Linezolid(100\%each), followed by Amoxicillin(76.9\%), Clindamycin(57.7\%), Erythromycin(53.8\%), Amikacin(50\%) and Azithromycin $(46.2 \%)$. Relative resistant patterns were noted in Gentamicin(76.9\%) and Cefoxitin(61.5\%). 16 isolates $(61.5 \%)$ out of 26 , were found to Methicillin resistant Staphylococcus aureus(MRSA), showing $100 \%$ sensistivity to Vancomycin and Linezolid. A study of the bacteriological profile and antibiotic resistance in a burn unit in France ${ }^{9,6}$ and Kuwait study done by Sanyal S.C et $\mathrm{al}^{9,7}$ established methicillinresistance rate of $68.1 \%$ and $66 \%$ respectively which is concordant with findings of the current study. Gramnegative bacilli showed high percentage of resistance to antibiotics like gentamicin and amikacin, ciprofloxacin, tobramycin, amoxicillin, cefotaxime and ceftriaxone. This alarming trend was seen for both Enterobacteriaceae group and for Pseudomonas species as seen in the study done by Manjula Mehta et al., ${ }^{4,8}$ This alarming rise of multidrug resistant isolates is probably due to empirical use of broad-spectrum antibiotics and non-adherence to hospital antibiotic policy. Therefore early detection of isolates is very important to prevent treatment failure as the time involved in isolation, identification and performing antibiotic sensitivity can take as long as 48 hours, during which a sub clinical infection could become a life threating illness. Moreover, because of the mixed infection in burn wounds, the potential virulence of one organism may enhance another organism growing alongside.

\section{Chart 1: Gender Distribution}

\section{Gender Distribution}

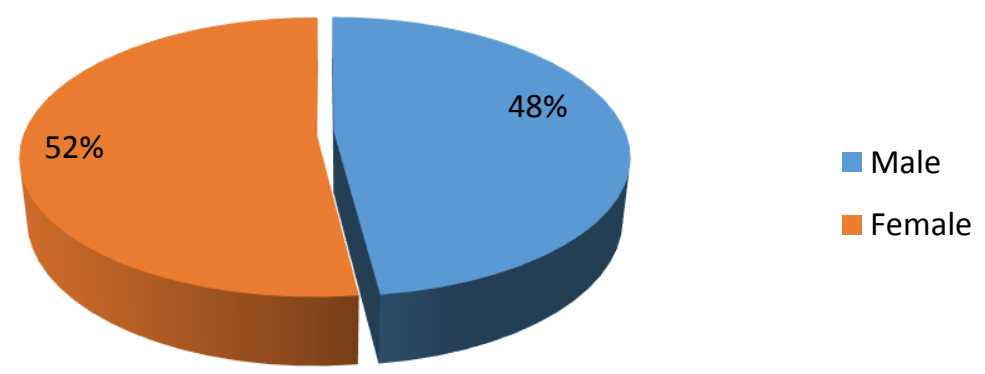

\section{Chart 2: MDR Organisms}

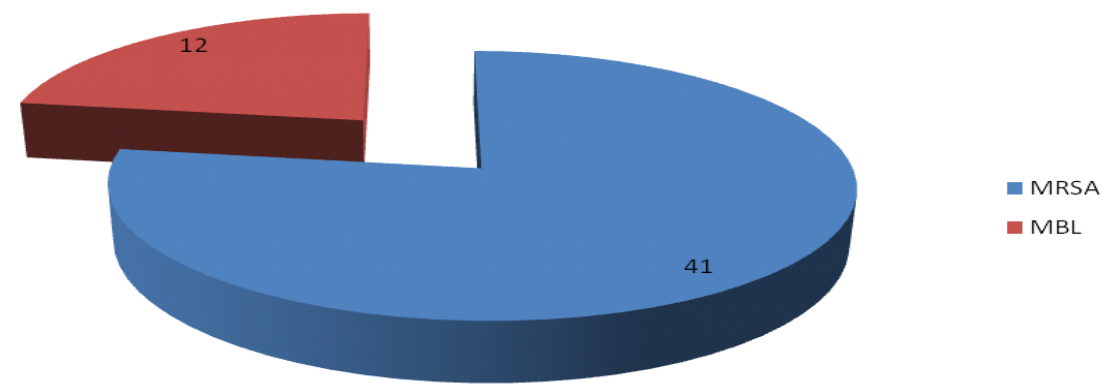


Graph 1: Distribution of patients with respect to various age groups

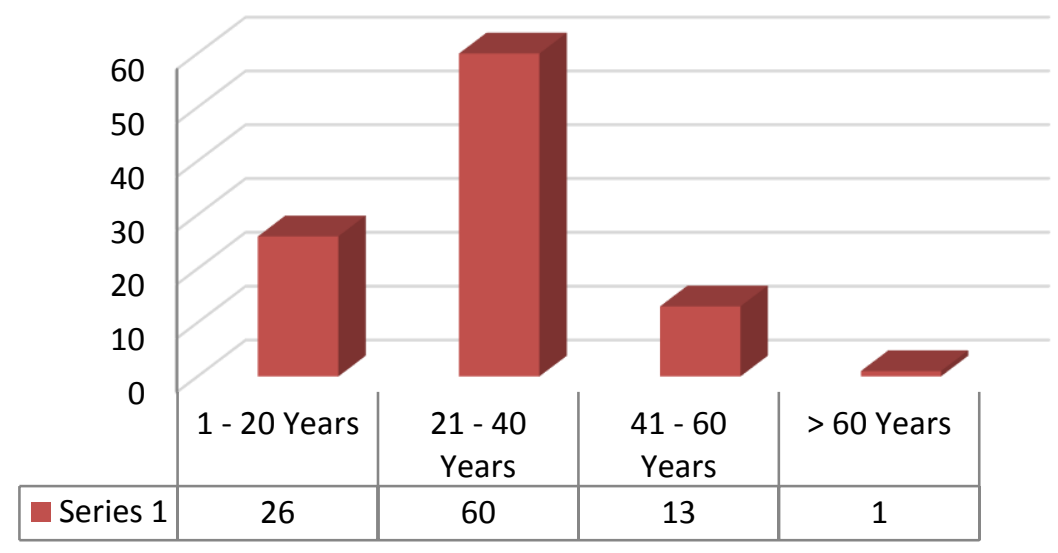

Graph 2: Prevalence of total body surface area burns

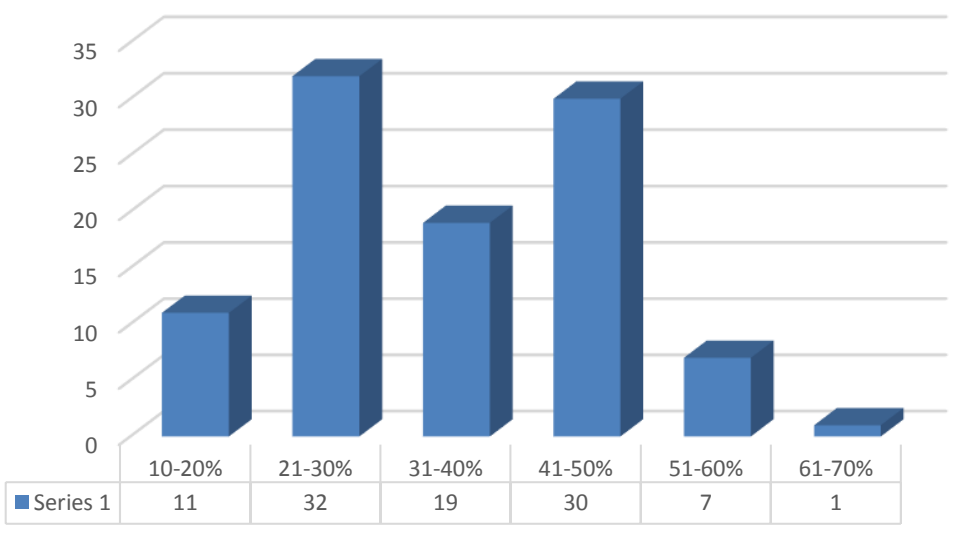

Graph 3: Differentiation of isolates by gram staining

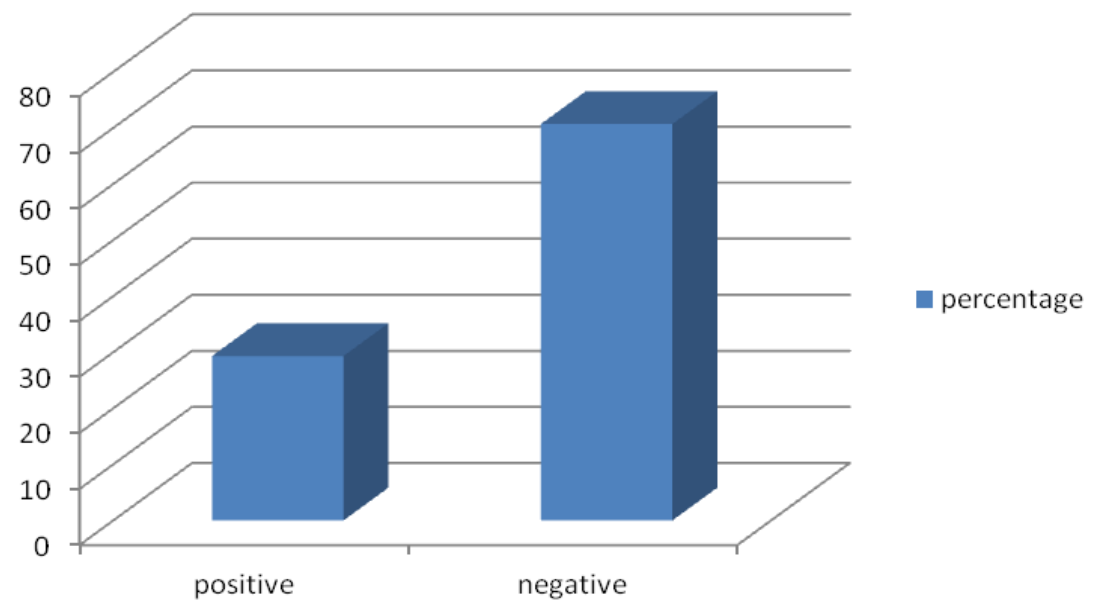




\section{Chart 3: Degree of burns}

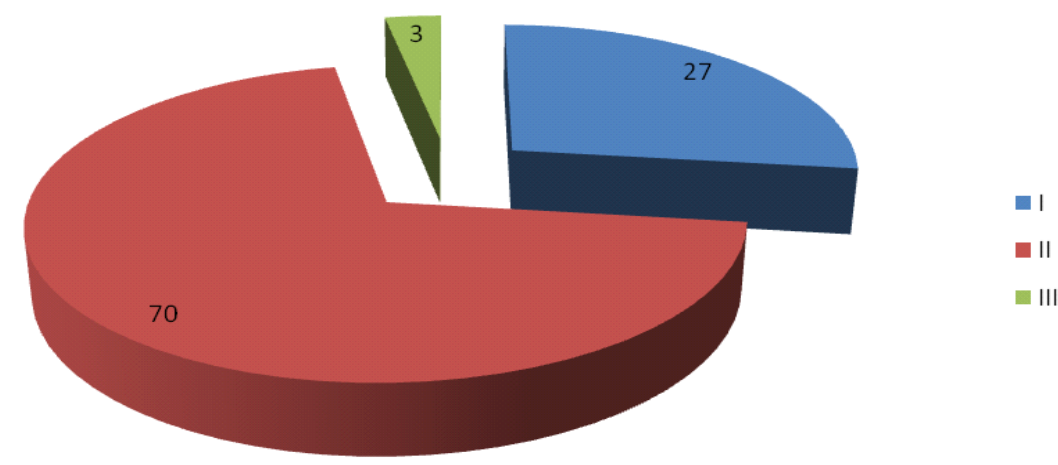

Chart 4: Prevalence of organisms from burnwound cultures

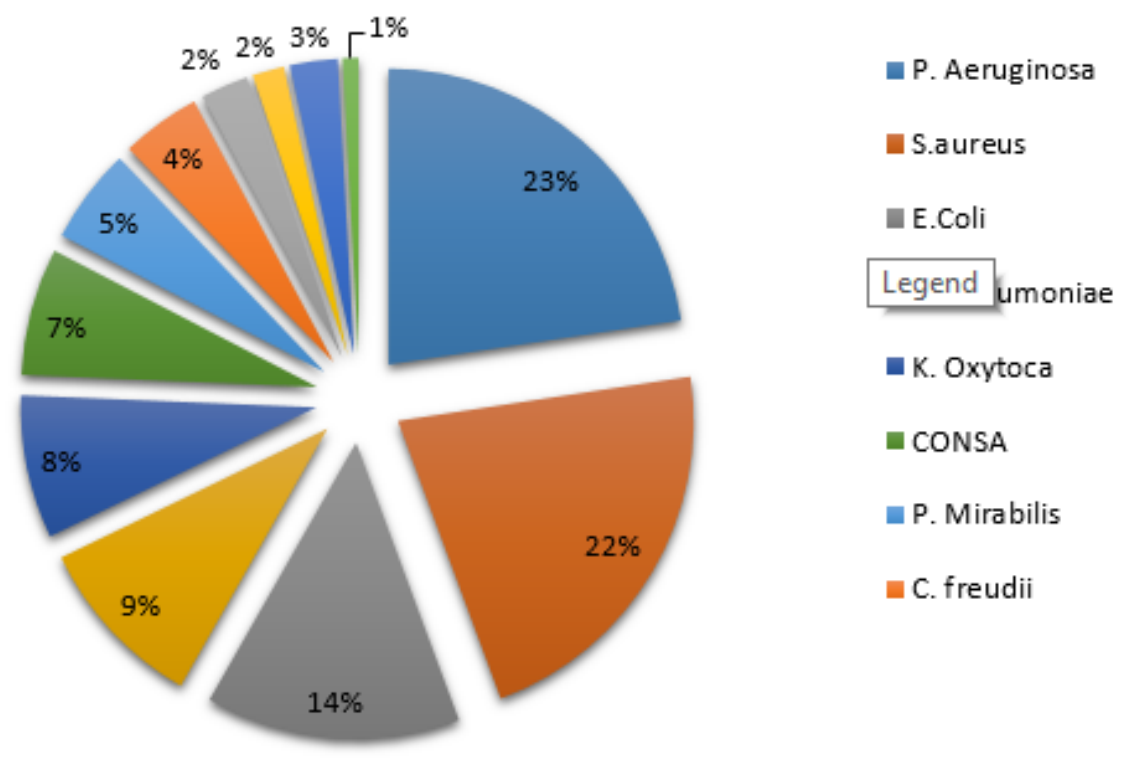

Graph 4: AMS of P. aeruginosa

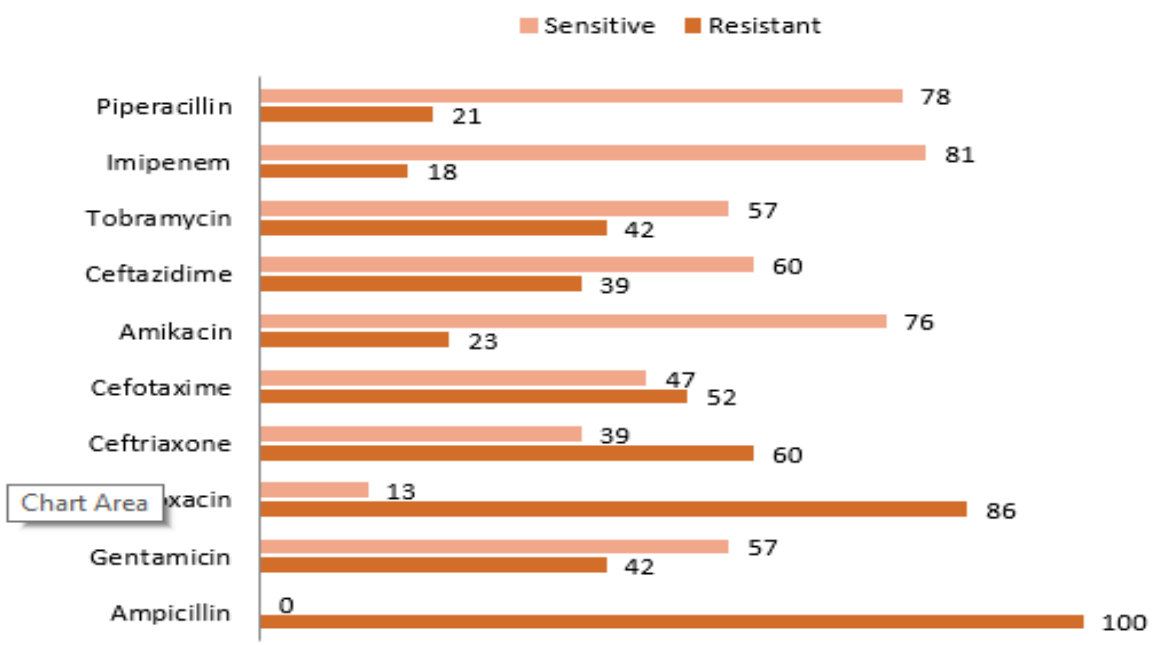




\section{Graph 5: AMS of Staphylococcus aureus}

\section{AMS of Staphylococcus aureus}

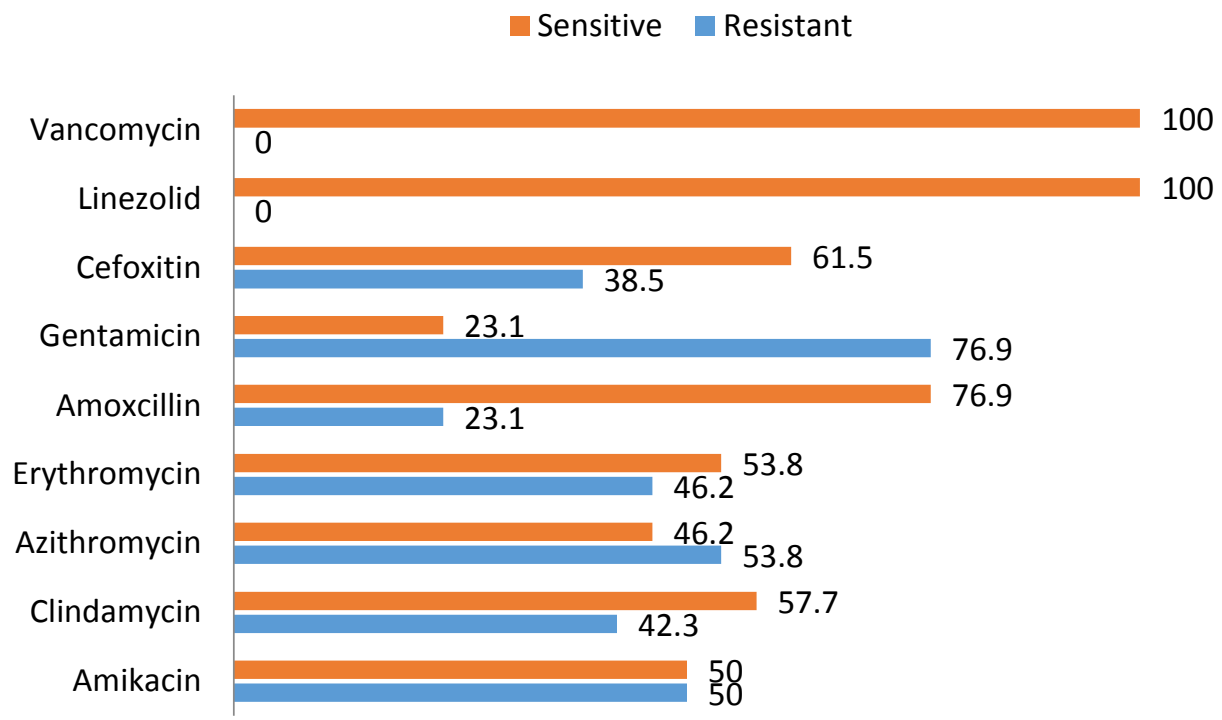

\section{Conclusion}

Pseudomonas is the main culprit in burn wound infections followed by S.aureus.

An important cause of cross infection in burn wards is overcrowding which must be avoided in order to control hospital acquired infections. Therefore, careful microbiological surveillance and in vitro testing before the start of antibiotic therapy and the use of restrictive antibiotic policy would help in prevention and treatment of MDR isolates in burn units thus reducing overall infection related morbidity and mortality .

\section{References}

1. Chauhan N, Kumar S, Sharma U. Profile of acute thermal burn admissions to the intensive care unit of a tertiary burn care center in India.Indian J Burns. 2012;20:68-71.

2. Hettiaratchy S, Dziewulski P.ABC of burns introduction.BMJ.2004;328:1366-8.

3. Gowri S, Vijaya N, Rajesh P.Epidemiological study of burn patients admitted in a District Hospital of North Karnataka, India.Indian J Burns. 2014;22:83-7.

4. Glen Mayhall.C.The Epidemiology of Burn Wound Infections: Then and Now.Clin Infec. Dis 2003;37:54350 .

5. O'Sullivan S.T. Immunosuppression following thermal injury: the pathogenesis of immunodysfunction. Br. J P Surg. 1997;50:615-23.

6. Edward J,Greenwood J.What's new in burn microbiology? James Laing Memorial Prize Essay 2000.Burns 2003;29:15-24.

7. Dennis G, Nathan P, Holder IA. Infected Surface Wound: an Experimental Model and Method for the Quantitation of Bacteria in Infected Tissues. Applied Microbiology.1972;23:509-14.
8. Mehta M, Dutta P, Gupta V. Bacterial isolates from burn wound infections and their antibiograms: A eight-year study.Indian J Plast Surg.2007;40:25-8.

9. Nicolle L, Bradley S, Colgan R et al.Infectious Diseases Society of America Guidelinesfor the Diagnosis and Treatment of Asymptomatic Bacteriuria in Adults.IDSA Guidelines for Asymptomatic Bacteriuria • CID 2005;40:643-54.

10. Liedburg NCF, Artz CP. The effects of bacteria on the take of split-skin thickness grafts in rabbits. Ann. Surg 1955; 142:92-4.

11. Hussein IA, Habib KA, Jassim KA. Bacterial Colonization of Burn Wounds. Baghdad Science Journal .2012;9:623-31

12. Mehta M, Dutta P, Gupta V.Bacterial isolates from burn wound infections and their antibiograms: A eight-year study.Indian J Plast Surg.2007;40:25-8.

13. Pandya NP, Prajapati SB, Mehta SJ, et al. Evaluation of various methods for detection of metallo- $\beta$-lactamase (MBL) production in gram negative bacilli.Int J Biol Med Res. 2011;2(3):775-7.

14. Batra A.K.Burn mortality:Recent trends and sociocultural determination in rural India.Burns 2003;29:270-5.

15. Akhtar, et al. Burn injury associated with co morbidities.Indian Journal of Burns. 2014;22:(1):51-4.

16. Chamania, et al. Burn wound infections and problems.Indian Journal of Burns.2012;20(1):18-22.

17. Azzopardi EA, Azzopardi E, Camilleri L, Villapalos J, Boyce DE, et al.Gram Negative Wound Infection in Hospitalised Adult Burn Patients- Systematic Review and Metanalysis-. Plos one.2014(9);4:1-7

18. Mohapatra S, Deb M, Agrawal K, Chopra S, Gaind R. Bacteriological profile of patients and environmental samples in burn intensive care unit: A pilot study from a tertiary care hospital. Indian J Burns 2014;22:62-6.

19. Bhardwaj SD, Sinha U. An epidemiological survey of burn injuries in rural area, Bhopal: A cross-sectional study.Indian J Burns 2012;20:62-5. 
20. Thabet L, Turki A, Ben Redjeb S, et al. Bacteriological profile and antibiotic resistance of bacteria isolates in a burn department. Tunis Med 2008;86:1051-4.

21. Sanyal S.C., Mokaddas E.M., Gang RX, Bang

R.L.Microbiology of Septicemia in Burn Patients. Annals of Burns and Fire Disasters.1998;XI:1-2.

22. Khan TS, Wani AH, Darzi MA, Bijli AH. Epidemiology of burn patients in a tertiary care hospital in Kashmir: A prospective study. Indian J Burns 2014;22:98-103.

How to cite this article: Qayoom $\mathrm{S}$, Jeer $\mathrm{M}$. Clinicomicrobiological profile of burn wound infections and antibiogram of the isolates in a Tertiary Care Hospital, Karnataka. Indian J Microbiol Res 2018;5(1):119-125. 